\title{
COMPUTATIONAL MODEL OF INTERSECTIONS REPRESENTATION AS APPARATUS OF CONTENTS MULTI-SCALAR, RETROSPECTIVE AND RESILIENT FOR THE RESEARCH OF CONNOTATIONS, SIMULATIONS AND SUSTAINABILITY IN THE LANDSCAPE ASSETS
}

\author{
Alessandro De Masi \\ Brera Academy of Fine Arts Milan, Department of Design and Applied Arts, Milan, Italy, alessandro.demasi@ unina.it, orcid.org/0000-0003-3433-2583
}

\begin{abstract}
KEY WORDS: Computational knowledge/Integration, Computational Intersections, Retrospective Intersections, Retrospective Comparison Classification, Multi-scalar Approach, Computational knowledge of digital intersection, Multidimensional knowledge in Process, Emerging Professional Profiles
\end{abstract}

\begin{abstract}
:
The research project's objective, based on defining a "Computational Model of Intersections Simulation/Representation" as complex digital cultural infrastructure and computational knowledge, acquires the identity characteristics of the Computational Representation (phenomenological, geometry, visual, resilience, scalability analytical-interpretative knowledge) to promote for landscape and Cultural Heritage $(\mathrm{CH})$ : a) of systematize environmental information and protective by intersections of resilience's categories in the "thematic systems of families and types" of the landscape; b) a model of Smart CH for Industry 4.0 - 2021. The same time favors: a) thematic and retrospective intersections between formal and interpretative layers for the return of information from a spatial and functional; b) innovation by introduction of professional figures of the contemporary digital. The methodology is articulated on following intersection models: Connotations, Composition, Development (combination of complex formal, structural and geometric factors); Morphogenesis (innovative concepts of transformations form), Sustainability. From the point of view the results and conclusion the study allowed to highlight: a) "network of multi-scalar discrete relationships" with sequences of digital models for the relative tessellation of the surfaces; b) "resilience measure" by "building a digital model of morphogenesis"; c) systematize environmental information and protective by intersections of resilience's categories in the "thematic systems of families and types" of the landscape; d) "operative and phenomenological reading" of the changing complexity of environmental reality with connections between the behavioural and physical structures of the place.
\end{abstract}

\section{INTRODUCTION}

\section{$1.1 \quad$ Objectives}

The research was born from the study conducted by the author as member the research group "Rural Vernacular Heritage 2007-11" - UNESCO Word Heritage Centre (WHC) - France (among whose objectives was the update 1972 World Heritage Convention's guidelines and supporting the Outstanding Universal Value); in addition, as, acting as adjunct professor at the Milan Polytechnic and the University of Rome III on the complexity of the processes of modification that took place in cultural and economic contexts with the simulcast description of information studies. The studies highlighted the need to integrate design strategies with interpretative/development synergies; in addition, the formation of languages and criteria based on integration/transformation to morphogenesis's design process. Therefore, the research project's objective is based on defining a "Computational Model of Intersections Simulation/Representation" as complex digital cultural infrastructure for landscape and Cultural Heritage $(\mathrm{CH})$. The study model acquires the identity characteristics by Computational Representation (De Masi, 2021), but that in fact favors potential, innovation and the introduction of professional figures of the contemporary digital; in addition, "resilience measure" by "building a digital model of morphogenesis". It is intended as: a) computational knowledge as integration between retrospective, natural formal, computer and interactive layers; b) "network of multi-scalar, discrete relationships with mosaic-like process for a surface continuity in Building Information Modeling (BIM) design. The infrastructure will be intended:

- Complex and Multi-scalar, for the study of the relationships and modifications in progress;

- Retrospective, for the comparison among models of interpretation relating to the modifications that have taken place;

- Resilient, due to the possibilities offered by the intersections of the study layers;

The study models, following the thematic and retrospective intersections, will return an uniformed information from a spatial, a functional, and a thematic point of view. In particular, it will allow to arrive, from parametric modelling to Big Data analysis; without neglecting the information models of augmented reality (AR) and, therefore, to promote a model of Smart Cultural Heritage for Industry 4.0 - 2021 (Figure 1). The creation of Smart Heritage aims at developing process of open innovation in the urban environment through sensitive analysis of new urban uses, territories stakes, prototypes, green technologies and operating process of users' collaboration (De Masi, 2014). With this premise, the digital cultural infrastructure will be able to:

A. reply: 
- at the National Recovery and Resilience Plan (PNRR): Next Generation Italia, the Urban and Territorial Regeneration regulations which aim to create applications of "Smart Cultural Heritage"; to the UNESCO directives (World Heritage Cultural Landscape, 2009; to the Convention for the Safeguarding of Intangible Cultural Heritage, 2003; to the Recommendation on the Historic Urban Landscape, 2011) and the ICOMOS directive (International Charter for the Conservation and Restoration of Monuments and Sites, 1964; to the Charter for Places of Cultural Significance - The Burra Charter, 2013);

- to experimentation with complex systems of representation in the range of digital modeling techniques;

B. allow:

- sequences of digital models for the reading of "compositional morphogenesis"; in addition subdivision and tessellation of design surfaces;

- the generation of complex integrated systems for the repetitive operations;

- learning the codes and languages of the current "digital design lexicon" of the computer age, that has actually replaced the "manual design lexicon";

- of systematize environmental information and protective by intersections of resilience's categories in the "thematic systems of families and types" of the landscape;

C. promote:

- a multi-scalar approach, of discretization of the topological surface and relative mosaicking;

- the evaluation in the digital language of information's exchange by generative algorithms of comparison with the diversified design choices;

- the inclusion, according to Cavalcoli, 2021 in the University Degree Programs of Architecture and Academies of Fine Arts, of emerging professional profiles such as: a) "Broad band architect", who works to bring the interactive web innovations back to TV by taking care of their contents in a smart way; b) "Cloud architect" based on the spread of "cloud computing" for the design and construction of scalable and resilient cloud environments; that is, capable of responding positively to change and the business needs of a company; c) "Data scientist", professional figures able to work with data, reading the numbers and interpreting them to achieve precise business goals; d) "Energy manager", a professional in support of top management to encourage the use of energy in the organization's structure by identifying the actions, interventions and procedures necessary to promote the rational use of energy; e) "Historic Building Manager", according to the Italian Ministry of Culture, in relation to management activities with economic operators, to the assignment of ordinary and extraordinary maintenance works; in addition to support for the various phases of the procedures through BIM software packages, plant design, data and communication networks;

- long-term regenerative synergies for empty spaces:

- "operative reading" to identify criteria and address lines in environmental insertion techniques. This will highlight the material culture of the place, the genius loci and to connect the constructive cultural traditions (De Masi 2018);

- phenomenological reading of the changing complexity of environmental reality with connections between the behavioural and physical structures of the place (Christian Norberg-Schulz - 1926-2000, Kevin Andrew Lynch 1918 1984) - (De Masi 2018);
- knowledge and communication of modification's processes of both the $\mathrm{CH}$ and the genius loci implemented for "categories of representation" (De Masi, 2018).

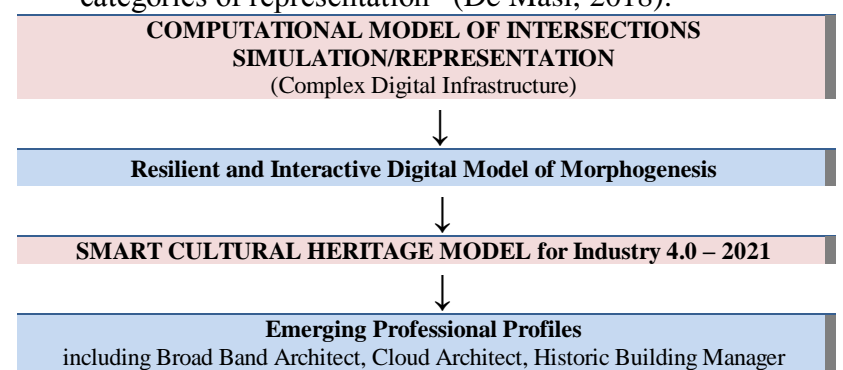

including Broad Band Architect, Cloud Architect, Historic Building Manager

Figure 1. Complex Digital Infrastructure Model.

\section{MAIN BODY}

\subsection{Research Methodology}

The methodology will be articulated on the following five layers of study (Figure 2):

\begin{tabular}{|c|c|c|c|}
\hline $\begin{array}{l}\text { IMC1 } \\
\text { Intersection Model of } \\
\text { Connotations based on } \\
\text { criteria of analysis, } \\
\text { evaluation of } \mathrm{CH} \text { assets } \\
\text { and spatial connections } \\
\text { with the urban space }\end{array}$ & \multicolumn{2}{|c|}{$\begin{array}{c}\text { IMC2 } \\
\text { Intersection Model of } \\
\text { the Composition, as } \\
\text { combination of creative } \\
\text { and generative factors of } \\
\text { the form }\end{array}$} & $\begin{array}{l}\text { IMD } \\
\text { Intersection Model of } \\
\text { the Development, as } \\
\text { combination of complex } \\
\text { formal, structural and } \\
\text { geometric factors }\end{array}$ \\
\hline \multicolumn{4}{|c|}{ ( } \\
\hline \multicolumn{2}{|c|}{$\begin{array}{c}\text { IMM } \\
\text { Intersection Model of the } \\
\text { Morphogenesis, as processes of } \\
\text { transformations form; Simultaneous } \\
\text { contrasts; dematerialized surfaces }\end{array}$} & \multicolumn{2}{|c|}{$\begin{array}{c}\text { IMS } \\
\text { Intersection Model of Sustainability, } \\
\text { as result of the procedures operating } \\
\text { in the category Interpretative Quality, } \\
\text { Internal Qualities, External Qualities, } \\
\text { Expressive Qualities (De Masi, } \\
\text { 2021). }\end{array}$} \\
\hline
\end{tabular}

Figure 2. Computational Model of Intersections Simulation/Representation.

\subsubsection{Intersection Model of Connotations (IMC1)}

The layer, through study apparatuses, will allow to define "Intersections models of the connotations of the Landscape and of the $\mathrm{CH}$ " by multidimensional disciplinary comparison (Figure 3); it is intended as:

- criteria of Heritage significance;

- knowledge model CH's state by means of a retrospective comparison among models of interpretation;

- individuation the fundamental spatial connections among the different constituent elements of the aggregate;

- anomalies identification and structural instability in order to choose the most suitable form of intervention architectural contest.

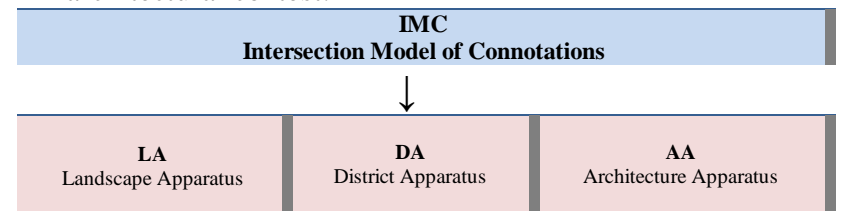

Figure 3. Intersection Model of Connotations (IMC).

Below are the study apparatus with the related sub-categories: LA. Landscape Apparatus

A. "Landscape's Interpretative Classification", intended as distinction between human and natural factors for identity, recognisability and visibility of the articulation of the landscape with the subcategories: 
IE. Identity's Elements, with the following layers: Physiotopes; Open Space; Anthropological Semiology (in order to define infrastructural, settlement, cultural, historical, architectural sign of arrangement, management of the historicized landscape and the articulation of the Urban and Rural Historical Landscapes); Absolute Visualisation (that allows the display of the great lines of the perceptible landscape) - (De Masi, 2020);

MC. Morphological's Characters through the study the forms of the ground, the distance and position of the observer according to Lynch and described by following subcategories: Visual Planes (succession of elements in the space); Visual Cones (distinguished by dimension, depth, orientation); Main reference points (useful elements for orientation and localization) - (De Masi, 2020); Main / visual emergencies; Margins / visual barriers; Elements of visual disturbance; Study of profiles; Chromaticity of volumes and predominant chromaticity; relationship of buildings to each other and to the environment (Standards and Guidelines for Architectural and Historical Investigations in Maryland, USA - SGAHI, 2019); Delimited Visual Scopes (limited and enclosed view) - (De Masi, 2015);

B. "Aggregations and Articulations of the Landscape's Classifications"(De Masi, 2008a, 2008b)

CAS. Characterizing Aggregation Systems and their exchanges with nature and anthropic reality;

PLS. Predominant Landscape Systems;

URHLA. Urban and Rural Historical Landscapes Articulation with the environmental mosaic;

RSPUIPS. Relations between sites and production, urban, infrastructural and physiographic systems of the Territorial Areas (De Masi, 2008a, 2008b);

A. Articulations with the following layers: Resistance and fragility of open and closed urban spaces, Fragmentation of green spaces, Permeability of built environments with green ones, Porosity of the anthropic environment, Environmental disorder, Integrity and connection of green spaces,Significant environmental attractors (Ferrara and Campioni, 1997);

SF. Sensitivity factors in order to evaluate: a relationship between manufacts and territorial context; geographic, geomorphological, and anthropic characteristics; accessibility (De Masi, 2008, 2015b);

\section{DA. District Apparatus}

AD. Appearance of the district during the time with achieved significance and any substantial modifications (SGAHI, 2019);

CD. Character of the district (residential, commercial, industrial, cultural) and types of buildings and structures (SGAHI, 2019);

CI. Condition and identity of the buildings, (including alterations, additions, restoration) - (SGAHI, 2019);

QD. Qualities of the district and open space such as parks, agricultural areas, vacant lots or ruins that is where relevant activities took place in history or prehistory (SGAHI, 2019);

\section{AA. Architecture Apparatus}

C. "Classification of the Significances" with subcategories: SS. Signifier/Signified that as has been acknowledged by Saussure (1915), the signifier and the signified are inseparably tied together, although the relationship is contextually and arbitrarily conventionalized (Durmus and Gur, 2011); Historic and Aesthetic (style, technical excellence, beauty, quality of design and execution), $\underline{\text { Scientific or Research, Social and Spiritual, Intrinsic }}$ (authenticity, extent/completeness, integrity, continuity of use/demonstration, corpus of evidence/study), Contextual: (rarity, representativeness /uniqueness, diversity, physical context, threat/fragility), Associative (historic interest and association, aesthetic attributes) - (Guidelines on $\mathrm{CH}$ of the European Union, Council of Europe, 2012);

IV. Intangible Values including appropriateness, ambiguity, modernism, visual power, intellectual elegance, timelessness, accumulated value, arbitrariness, marketing (Vignelli, 2012); TV. Tangible Values including relationship between bodies, contrasts of size, scale, surfaces, color, sequence, identity and diversity (Vignelli, 2012);

AA. Aesthetic attributes (Vignelli, 2012);

F. Function/form, plan/volume, marginality/centrality, served/servant, fixed/flexible, stable/flowing, repetitive/unique, fit/misfit, discovered/invented, concept/vision, material/transcendental, concept/sign (Durmus and Gur, 2011);

IQ. Interpretative Quality with the subcategories: Identity and diversity; Continuity and modification of the identity;

D. "Retrospective Comparison Classification", as inventory of Historic Monuments to provide information and data record for a wide range of historic buildings/monuments. The layer were divided into the following subcategories (additional information has been obtained by the Italian Ministry of Heritage and Cultural Activities's programs)- (De Masi, 2015):

IBL. Identification with the following layers: Identification of building location related to a risk area and to a surrounding urban context; $\underline{\mathrm{CH}}$ 's historical evolution as a sequence of the phases of building transformation; Identification of the building's structural elements, of the construction techniques; The identification of materials, their degradation; Knowledge of subsoil and foundation structures, focusing on the changes that occurred over time and the relative instability; Constructive aspect with sub-categories related to the survey of constructive and decorative details (scales 1:20 and 1:5); walls relief with respect to materials and finishes, plasters and coatings; Type of building, intended use, pre-existing and current; Current technological aspects; Classification of identity and contemporary signs from the different elements contained in the sub-categories; Registry identification for a three-parametric evaluation: denomination, toponymy and land registry data - SGAHI, 2019);

SF. Sensitivity factors in order to evaluate: a) a relationship between manufacts and territorial context; b) geographic, geomorphological and anthropic characteristics; c) accessibility (De Masi, 2018);

ME. Morphology elements in order to identify structural elements, inspectionability, building technique, materials and elements of historic and/or artistic value;

PS. Preservation State;

E. "Survey Guidelines Classification", as inventory of survey for historic buildings/monuments to allow document information and to identify, preserve, protect and sustain collective architectural heritage (De Masi, 2015) - (Figure 4). Additional information has been obtained by the Italian Ministry of Heritage and Cultural Activities's programs; in particular the layer is articulated on:

HDR. Historical documentation research of archival sources with attention to the relationship between work and context;

PS. Photographic survey of the different facades, of the roof, of the entrance, and of the staircase; 

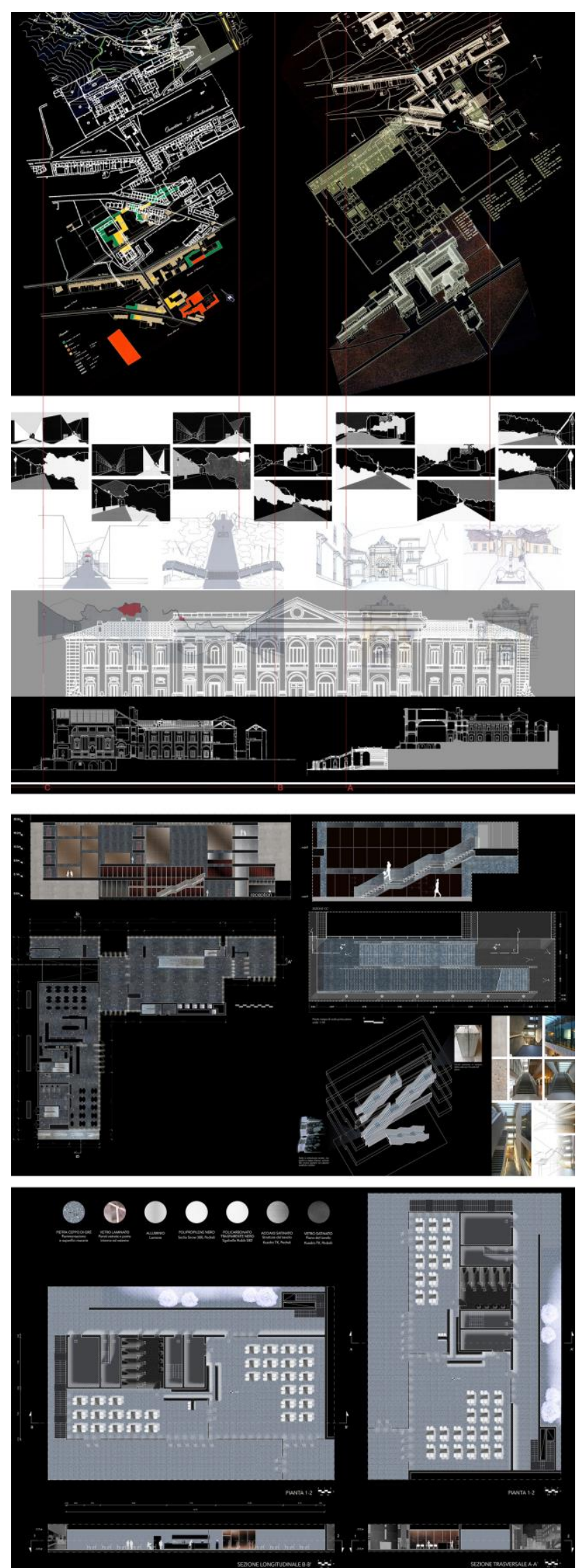

Figure 4. Belvedere di San Leucio (Caserta); New Bocconi University, Milan (2008) - Polytechnic of Milan. IMC1,

Landscape Apparatus: more in-depth knowledge of the complex structure by Morphological's Characters; Architecture Apparatus: Survey (A. De Masi).
RI. Registry identification for a three-parametric evaluation: denomination, toponymy and land registry data. (SGAHI, 2019);

CF. Current formal aspect with the subcategories related to dimensional factors of the facade height and the type of hedge; study of the geometrical relationship between full and empty areas; horizontal and vertical alignments and study of the openings axle spacing with an indication of the pace; geometry and proportion for architectural details and for the facade significant profiles; dimensional scale survey of the compartment; dimensional relief with drafting of the plan, sections and elevations (scale1:50); Survey with drafting of the architectural plan, sections and elevations (scales 1:50, 1:25). Specifically, this phase will focus on: the typological transformation system; the formation of aggregated interior spaces; spatial relationships of the individual cell walls; regularity and modularity at the different floors; the walls alignment, rotations and intersections of the walls axes; Shapes and positions of the openings in the walls (De Masi, 2015);

OI. Other issues include: Deterioration of the property's historic integrity; Date of restoration; the historical basis for the work done; the amount of remaining historic material and replacement material; The effect of the work on the property's historic integrity; The historic appearance and current condition of natural features (SGAHI, 2019); landscape features and vegetation that characterized the property; the historic appearance and current condition of both man-made features and natural features throughout the area; Land uses, features, and vegetation that characterized the roadway (SGAHI, 2019).

SA. Surfaces analysis through a chromatic survey with regard to the type of color, the color shade, and the texture of the material. In this case, it is essential to identify the percentage of the existing colors, their classification code, the areas of degradation, and the amount of degraded surface.

\subsubsection{Intersection Model of the Composition (IMC2)}

The layer is intended as combination of creative and generative factors of the form and in particular study of the following categories (Figure 5):

- Formal aspects: verification of the shape's characteristics;

- Internal Qualities: Connection, Inclusion, Division of the mutation; Disintegration and reconstruction of the form; Evaluation, Hierarchy;

- External Qualities: Cultural models and relational structures.
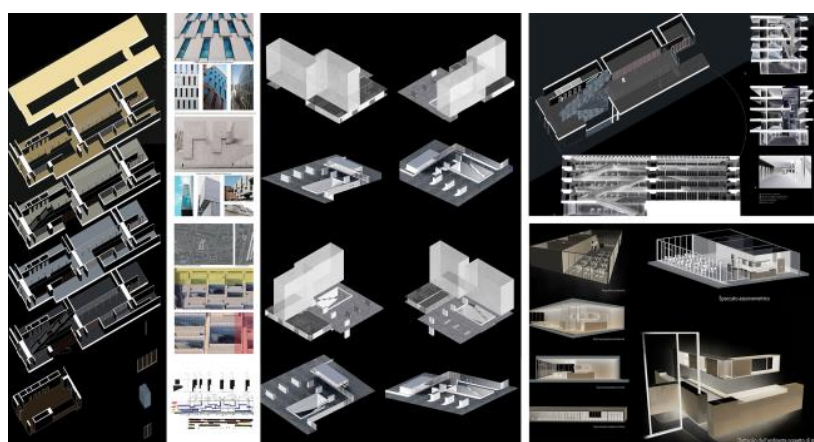

Figure 5. New Bocconi University, Milan (2008). IMC2: Formal Aspects and Internal Qualities. School of Design, Polytechnic of Milan (A. De Masi) 


\subsubsection{Intersection Model of the Development (IMD)}

The layer is intended as intersection's combination of complex formal, structural and geometric factors, fundamental for the development of the form; it focuses on use of the mathematical sequences to produce continuous and uninterrupted surfaces; the volumes, of some architectures in Milan (Giangiacomo Feltrinelli Foundation, Herzog \& de Meuron; Penthouse one11, Hadid Architects), are not only formally and spatially integrated but also technologically thanks to electronically solicited sound algorithms. The continuous surface, for example, shows incredible similarities with its wire-frame version, as it refers to an exact geometric reproduction and, at the same time, it is a direct expression of the virtual mapping of the surface (De Masi, 2011) - (Figure 6). This process, where digital information is of course related to manufacturing methods as directly joined to the mold of the object, was performed to achieve a new aesthetic with a new form of architectonic ornamentation (De Masi, 2011). New object forms were created as well as a new architecture contaminated by the provocative possibilities of the new generation of software abusing the concept of efficiency and production to invade the territories of thought and space planning (De Masi, 2011, 2020).
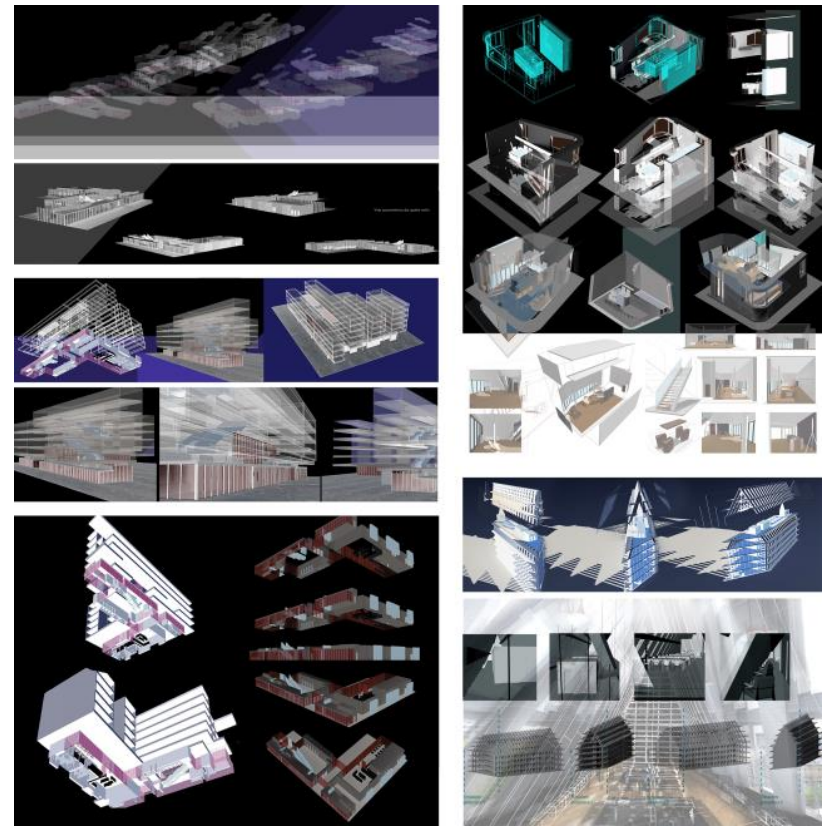

Figure 6. New Bocconi University headquarters in Milan Grafton building (2008); Giangiacomo Feltrinelli Foundation, Milan, Herzog \& de Meuron; Penthouse one-11, Milan (Hadid Architects). IMD: Continuous and uninterrupted surfaces. School of Design, Polytechnic of Milan, Brera Academy of Fine Arts Milan (A. De Masi)

\subsubsection{Intersection Model of the Morphogenesis (IMM)}

The layer is intended as process of transformations form; in particular, processes intersection's combination for the research of: Complex Geometries (complex system and formal organization); Digital Prototype intended as combination of Digital Invariant (based on innovative design choices - themes and details) and Digital Ambiguities (result of the interpretations on simultaneous contrasts and dematerialized surfaces). The layer is based on: a) innovative concepts of reading of the "compositional morphogenesis" intended as "sequences of digital models" by parametric shapes and acquisition of studies on topological geometry, isomorphic surfaces, metamorphic architecture and genetic algorithms; b) codes and languages of the current "digital design lexicon". It will allow to obtain a "computational knowledge" based on:

- a "multi-dimensional knowledge in process" on different levels of study for performative purposes; it will be understood as a "knowledge representation" in its historical evolution between object and landscape; taking into account the constraints of the materials, the specificity of the urban environment, the shared space and the qualities of the external system;

- spatial compartment of objects, decomposition of the lineforce, balance between transparent volume, force interpenetration, geometric construction and algorithm;

- categories of digital intersection related to design process: technological and representative categories;

- a control of "complex geometries" in the digital design field with the simulation the design data, that in fact has taken over the representation;

- overcoming of the dichotomy between designed shape and realized forms.

As shown above the layer, the layer provides, for the purposes of innovation in the disciplinary field of morphogenesis the multidimensional comparison between the following study categories:

Digital Apparatus

A. "Digital Classifications" with sub-categories:

CG. Complex Geometry;

DP. Digital Prototype: Digital Invariant and Digital Ambiguities;

\section{Visual Apparatus}

B. "Computational Representation's Classifications" based on:

G. Geometry, for the study of shapes, of geometric matrices and data search relative at topography, photogrammetry, matching the model and the point cloud;

V. Visual, through information models: Evolutionary knowledge (in their historical becoming) with simulation lexicon (reconstruction process and digital restitution of horizontal and vertical elements, overlapping data (De Masi, 2020). In particular, description of the design matrices and phenomenological reading of the changing complexity of environmental reality; Non-Evolutionary knowledge (at a given moment, abstracting from their evolution over time) without lexicon simulation (De Masi, 2020) and description of the evolutionary matrices;

S. Scalability, to configure multi-scale models of the visual field (De Masi, 2021) - (Figures 7,8).

In this sense, the simulation / representative processes of the morphogenesis, that are also planning, actually constitute the constructive and implementation basis of the project itself. The objects from the mechanical technology are directly attributable to the forms that generated them, while those produced with digital technology result from a sequence of numbers that makes them easily changeable. The change in the number series generates a digital mutation of the object and of its representation thus producing similar but different forms, mediated by complex processes of interfaces corresponding to networks and to the utilized computer systems. Therefore, the building process is an expression of the unpredictability or intentionality of the algorithmic process and, only in part, of the will of the operator as in the images presented. 

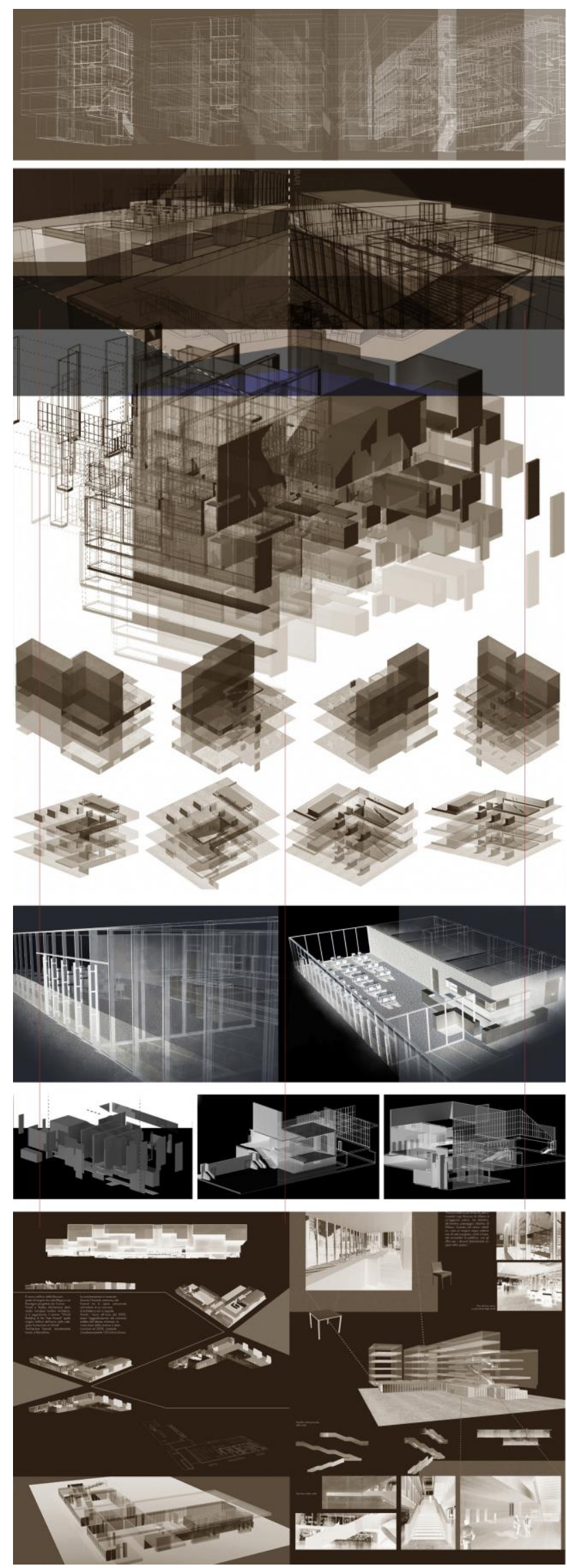

Figure 7. New Bocconi University, Milan - Grafton building (2008). IMM: Digital and Visual Apparatus. Brera Academy of Fine Arts Milan and II School of Architecture, Polytechnic of Milan (A. De Masi).

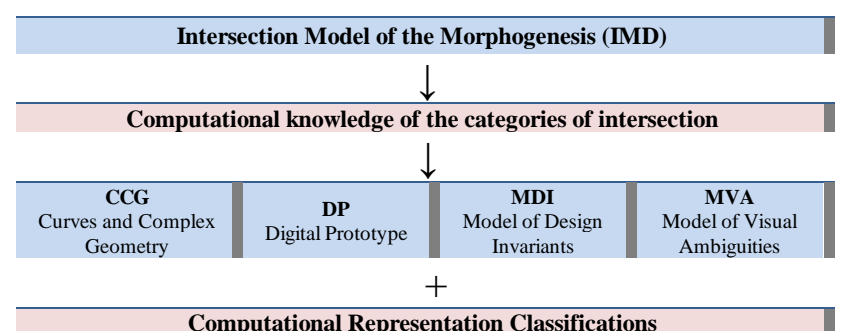

Computational Representation Classifications

Phenomenological + Geometry + Visual + Resilience

Figure 8. Intersection Model of the Morphogenesis Structuring.

As the digital transformation is not always controllable, some technologies have been designed to counter this endless process of transformation and crystallize the desired visual image on which to produce identical images (De Masi, 2011). The structural variability of digital technology produces significant changes in architectural forms and, more generally, in the outward and visible form of our built environment. The designers, therefore, have focused on the formal possibilities offered by continuous functions generated by the mathematical algorithms with which fluid shapes, curves and complex geometry are produced (in particular topological geometry), materialized in three dimensions by file-to-factory technologies (stereolithography, rapid prototyping, and other tools for numerically controlled production) - (De Masi, 2011). The material construction of a prototype takes place when the process is blocked by isolating the selected snapshot from the sequence to which it belongs. What follows is the threedimensional materialization and the production of a single piece, while the production of a sequence becomes the mass production of unique pieces and it is very different from the reproduction in a series of identical pieces of the assembly. This is the new frontier of "non-Standard" representation ("mass customization").The numerical production of nonstandard series revolutionizes the concept of reproducibility we were used to by the mechanical culture. The reproducibility, therefore, depends on an algorithm re-proposing different but similar forms, of a non-standard series which have a common condition: the similar forms are recognizable because they descend from a unique algorithmic matrix. The digital culture meets the problem of the recognition of forms by referring to the normative aspects of similarity and not to the visual aspects with which you proceed to the identification of equal forms in the Fordist process (standard). (De Masi, 2011). In the picture just described, the characters of formal organization, delivery information and transformation of the form play a central role in the constitution of the form. They are perfectly linked, unlike modern and classic design processes, above all to external stresses to define a constant updating and generation of shapes in the language of the layers. The constant remodeling of space is a characteristic for the recognition of models in the language of computational representation/simulation; the formal generation process will be understood as integration of digital intersections in constant evolution. This for performative purposes through representation models for architecture and Urban Landscape in the simulcast description of information.

\subsubsection{Intersection Model of Sustainability (IMS)}

The layer is intended as:

- synergistic process between scientific research skills and the market; 
- aggregation of territorial development strategies;

- domain of collective memory assets;

- Visual representation model of spaces and contents with simultaneous description of information for the architecture and urban landscape;

and based on the following four criteria:

A. Strengthening of Cultural Identity

RPP. Regeneration of historical and productive landscapes; SCH. Smart Cultural Heritage;

B. Environmental Sustainability

SM. Sustainable Mobility;

ROP. Renaturalization of Open Spaces;

UF. Urban Fragmentation;

\section{Network of Professional Skills}

NS. Network of skills, enhancement and safeguard spinoffs for an eco-sustainable development model;

\section{Cultural - Tourist - Economic Development}

NA. Nodal Areas, areas where exchange processes between the urban environment, social culture and civil society are formalized (De Masi, 2008a, 2008b);

DT. Macro and Micro territorial districts, representative of the economic, social and architectural development of the environment and Landscape Areas (De Masi, 2008a, 2008b). This in order to establish a presence cultural - management interface with technical bodies, cultural institutions, universities;

DL. Different types of Sensitivity of Landscapes, through the introduction of the "Levels of Sensitivity"; they are called "trend threshold" of the anthropic territory. This is to facilitate the process of interchange between the cultural tourist - archaeological - urban - productive - landscape systems (present within the Districts) and the neighboring territory in significant areas called "critical nodes". All this within rural and urban environments in order to verify a constant dialectical relationship with modern urbanism and promote places of "characterizing attraction" (De Masi, 2008a, 2008b) - (Figure 9)

\section{Result and Conclusion}

The paper describes a research project, based on defining a "Computational Model of Intersections Simulation/ Representation" as complex digital cultural infrastructure and computational knowledge for landscape and Cultural Heritage $(\mathrm{CH})$ and understood as: a) systematize environmental information and protective by intersections of resilience's categories in the "thematic systems of families and types" of the landscape; b) model of Smart CH for Industry 4.0 - 2021; c) thematic and retrospective intersections between formal and interpretative layers for the return of information from a spatial and functional; d) innovation by introduction of professional figures of the contemporary digital. From the point of view the results and conclusion the study allowed to highlight: a) "network of multi-scalar discrete relationships with sequences of digital models for the relative tessellation of the surfaces; $b$ ) "resilience measure" by "building a digital model of morphogenesis"; c) systematize environmental information and protective by intersections of resilience's categories in the "thematic systems of families and types" of the landscape; d) "operative and phenomenological reading" of the changing complexity of environmental reality with connections between the behavioural and physical structures of the place.
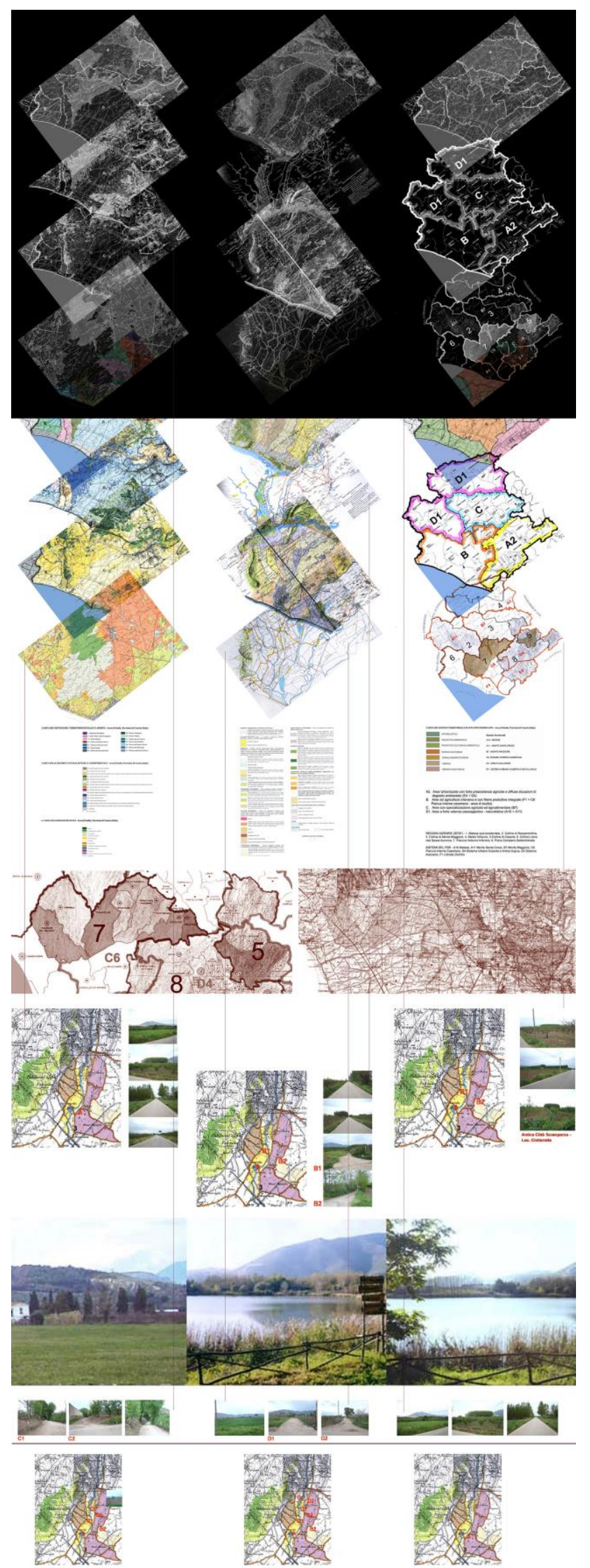

Figure 9. Municipalities of the Alto Casertano area. IMS, intersection between Landscape Apparatuses (Interpretative and Articulations classification) for the Strengthening of Cultural Identity and to promote Cultural - Tourist - Economic Development (A. De Masi). 


\section{REFERENCE}

Cameron, F.; Kenderdine, S., 2007: Theorizing Digital Cultural Heritage: A Critical Discourse, MIT Press: Cambridge, MA, USA.

Carpo, M., 2004: Ten Years of Folding, prefazione alla riedizione, in Folding in Architecture, by G. Lynn, London.

Cavalcoli, D., 2021: Lavoro, chi sono gli «introvabili»: i 6 profili che tutte le aziende vorrebbero assumere. In Corriere della Sera, L'Economia, RCS MediaGroup.

De Masi, A., 2021: Digital Documentation's Ontology: Contemporary Digital Representation as Express and Shared Models of Regeneration and Resilience in the Platform BIM/Contaminated Hybrid Representation. Int. Arch. Photogramm. Remote Sens. Spatial Inf. Sci.

De Masi, A., 2020: From Computational De-Morphogenesis to Contaminated Representation for the Contemporary Digital Tectonics and Lexicon. Vulnerability and Resilience of the Generative Representation in the Web-Oriented Platform BIM/GD. In IEE BigData 2020, Volume 1, pp. 1884-1893, IEEE, 10-13 dicembre 2020, Atlanta, GA, USA, DOI:10.1109/ BigData50022.2020.9377781.

De Masi, A., 2018: Eco Sustainable Graphic Heritage Drawing for a Contemporary Territories Learning and the creating Smart Cities. In Progress in Cultural Heritage: Documentation, Preservation, and Protection. EuroMed 2018. Lecture Notes in Computer Science, vol 11197, pp 262-270, Springer, Cham.

De Masi, A., 2015: Survey Guidelines and its Reading Criteria for Monitoring and Transmitting Cultural Heritage Values. Int. Arch. Photogramm. Remote Sens. Spatial Inf. Sci., Volume XL-5/W7, pp. 113-121, 2015, doi:10.5194/isprsarchives-XL-5W7-113-2015.

De Masi, A., 2014: Smart Cultural Heritage and Open Source as Regeneration of Historical Centers: Fruition, Conservation and Preservation in Current Methods of 2D/3D Digitization and 3D Modelling. In IEEE International Conference on Big Data and Cloud Computing (BdCloud), Sydney, Australia, pp. 729-736, doi:10.1109/BDCloud.2014.114. ISBN 978-1-47996719-3.

De Masi, A., 2011: Architectural Models and Complex Geometries. In The International Journal of the Image, Los Angeles, University of California, USA, Volume 1, Number 3, Common Ground. ISSN 2154-8560.

De Masi, 2009: Campania Felix (Italy), Cultural Landscape and rural environments governance (October 2008). $1^{\circ}$ Report of International Team Research - UNESCO World Heritage Centre (Paris), "Rural Vernacular Heritage" 2007-2011". In UNESCO World Heritage Centre (France). I.S.S.N. 18871658.

De Masi, A., 2008a: Campania (Italy), Cultural Landscape and Rural Environment Governance. In Proceedings of Heritage, Barcelos: Green Lines Istituto, vol.2, pp.607-617.
De Masi, A., 2008b: Campania Felix (Italy), Cultural Landscape and Governance of Rural Environments. Technical Report 1. Research Team: "Rural Vernacular Heritage 200711", UNESCO World Heritage Centre (France).

Durmus, S., Gur, S.O., 2011: Methodology of deconstruction in architectural education. In WCES, Procedia - Social and Behavioral Sciences, Elsevier doi: 10.1016/j.sbspro.2011.03.335.

Ferrara, G., Campioni, G., 1997: Tutela della Naturalità Diffusa, Pianificazione degli Spazi Aperti e Crescita Metropolitana, Il verde Editoriale s.r.l., Milano.

Guidelines on Cultural Heritage Technical Tools for Heritage Conservation and Management, European Union, Council of Europe, September 2012.

Kolarevic, B., 2000: Digital Morphogenesis and Computational Architectures. In $4^{\circ}$ SIGraDI 2000, Rio de Janeiro.

Lynn, G., 1993: Architectural Curvilinearity: The Folded, the Pliant and the Supple. AD Profile 102: Folding in Architecture. London: Academy Editions.

Lynn, G., 1998: Animate Form. Princeton: Princeton Architectural Press.

Menges, A., 2010: Form Generation and Materialization at the Transition from Computer-aided to Computational Design, Detail (English Edition), Vol. 2010 No. 04, pp. 330-335.

Menges, A., 2007, Computational Morphogenesis, Proceedings of the Third International Conference of the Arab Society for Computer Aided Architectural Design (ASCAD), Alexandria (Egypt) 28-30 November 2007, pp. 725-744.

Menges, A., 2011, Sistemi semplici - Capacità complesse. Processi integrativi di morfogenesi computazionale in architettura, TECHNE 02.

Italian Ministry of Heritage and Cultural Activities's programs

Pescarin, S., 2016: Digital Heritage into Practice. SCIRES-IT, $6,1-4$.

Silberman, N., 2016: Heritage places: Evolving conceptions and changing forms. In A Companion to Heritage Studies; Logan, W., Craith, M.N., Kockel, U., Eds.; Wiley-Blackwell: London, UK.

Standards and Guidelines for Architectural and Historical Investigations in Maryland (SGAHI), Maryland Historical Trust Maryland Department of Planning, 2019 Maryland, USA

Vignelli, M., 2012: Il Canone Vignelli, Postmedia Books.

Wing, J., 2006: Computational Thinking - Communications of the ACM vol. $49, n^{\circ} 3$, pp. 33-35.

Zellner, P., 1999: Hybrid Space: New Forms in Digital Architecture. New York: Rizzoli International Publications. 